\title{
SUPERPLASTIC FORMING SIMULATION OF RF DETECTOR FOILS
}

\author{
Q.H.C. Snippe ${ }^{1 *}$ V.T. Meinders ${ }^{2}$ \\ ${ }^{1}$ National Institute for Subatomic Physics (Nikhef), Engineering Department, Amsterdam (NL) \\ ${ }^{2}$ University of Twente, Department of Mechanical Engineering, Enschede (NL)
}

\begin{abstract}
Complex-shaped sheet products, such as R(adio) F(requency) shieldings sheets, used in a subatomic particle detector, can be manufactured by superplastic forming. To predict whether a formed sheet is resistant against gas leakage, FE simulations are used, involving a user-defined material model. This model incorporates an initial flow stress, including strain rate hardening. It also involves strain hardening and softening, the latter because of void formation and growth inside the material. Also, a pressure-dependency is built in; an applied hydrostatic pressure during the forming process postpones void formation. The material model is constructed in pursuance of the results of uniaxial and biaxial experiments.
\end{abstract}

KEYWORDS: Superplasticity, Material modelling, Forming simulations

\section{INTRODUCTION}

RF detector foils are used to separate two vacuum spaces inside a particle accelerator setup: the detector vacuum and the accelerator beam vacuum. This separation requires strict demands on the leak tightness of these RF foils. On the other hand, these foils should be as thin as possible, in order to prevent disturbance of particle tracks traversing such a foil.

A method to manufacture these foils is by means of Superplastic Forming (SPF). This forming process is characterised by the high-temperature pressing of a sheet in a one-sided die by means of gas pressure, such that the strain rate is very low. Failure of the material in these conditions is caused by the coalescence of internal voids, nucleated during the increasing strain. Applying a backpressure in the forming process postpones the nucleation and growth of the internal voids, which has a beneficial effect on the gas leakage of the formed sheet.

To include a specific flow behaviour (Hosford) and this pressure-dependent behaviour, a user-defined material has been built to be used in ABAQUS/Standard.

This article describes the necessary ingredients of this user-defined material model. Section 2 explains the phenomenon of superplasticity, focused on the mechanical properties. The user-defined material model is described in Section 3. With this model, the superplastic forming process can be simulated. The verification and simulation is the subject of Section 4.

\section{SUPERPLASTICITY}

A material behaves superplastically if it can attain very high plastic strains. It is provided that the material is subject to an elevated temperature (about $500{ }^{\circ} \mathrm{C}$ for superplastic aluminium alloys) and a limited strain rate (in the

*PO Box 41882, +31 205922105, csnippe@ nikhef.nl order of $10^{-3} \mathrm{~s}^{-1}$ ). These materials are very sensitive to changes in strain rate.

This section focuses on the mechanical behaviour of ALNOVI-1, an AA 5083-based superplastic aluminium alloy, manufactured by Furukawa Sky Aluminium, Ltd. The initial flow stress is strongly dependent on the equivalent plastic strain rate. Increasing the plastic strain will result in hardening and, due to cavity formation, eventually in softening of the material. Because the application of an external backpressure during forming influences the cavity formation and growth, the void volume fraction $\xi$ is incorporated in the determination of the flow stress.

\subsection{INITIAL FLOW STRESS}

The initial flow stress of superplastic materials is strongly dependent on the equivalent plastic strain rate. Vasin [1] demonstrates that this flow stress can be described as a so-called Universal Superplastic Curve. This curve has a sigmoidal shape when the logarithm of strain rate and flow stress are plotted against eachother, see Figure 1. Optimal superplastic behaviour is reached at the strain rate at which the highest slope in the curve occurs. The slope is the strain rate sensitivity $m$, which is a bell-shaped curve. Typical maximum values of $m$ for superplastic materials are in the range of 0.5 to 0.7 .

A general equation for a sigmoidal curve can be expressed by means of a set of four parameters, $a, b, c$ and $d$, according to Equation (1)

$$
\log \sigma_{\mathrm{y} 0}=\frac{1}{a+b \exp \left(c \log \dot{\varepsilon}^{\mathrm{p}}\right)}+d
$$

Tensile experiments are necessary to extract the values for these four parameters, in this case resulting in the initial flow behaviour of ALNOVI-1. The optimal temperature where the highest plastic strains were obtained in the experiments, is $520{ }^{\circ} \mathrm{C}$. The output of the experiments, in 


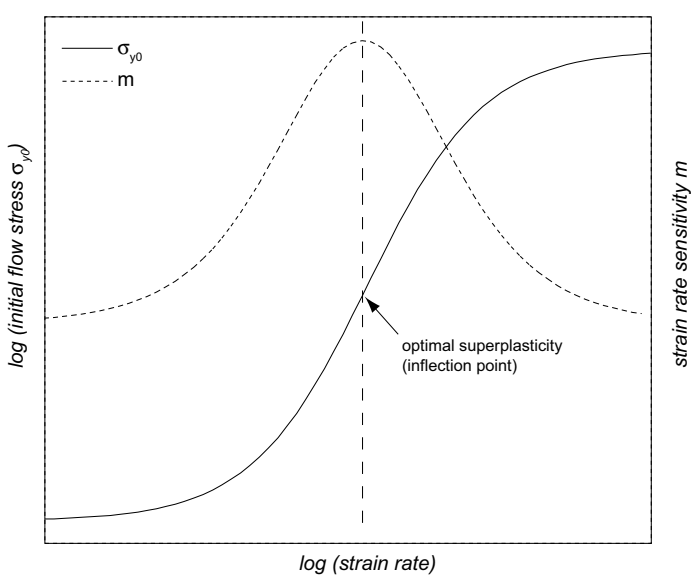

Figure 1: Sigmoidal shape of the initial flow stress and the strain rate sensitivity as function of the strain rate

terms of force-displacement relationships, was translated into sets of stress-strain curves. The initial value of the flow stresses at different strain rates were fitted to Eq.(1), see Table 1.

Table 1: Parameter values of $a, b, c$ and $d$ for ALNOVI-1

\begin{tabular}{cccc}
\hline$a$ & $b$ & $c$ & $d$ \\
\hline 1.4446 & $3.5633 \mathrm{E}-5$ & -3.8901 & 6.4332 \\
\hline
\end{tabular}

The maximum strain rate sensitivity $m$ is reached at a value of about $1.2 \cdot 10^{-3} \mathrm{~s}^{-1}$, the strain rate sensitivity at this point is 0.61 .

\subsection{STRAIN HARDENING}

The mechanism of superplastic deformation is different from conventional plastic deformation. The increase of plastic strain exhibits itself by the sliding of grains past eachother, instead of grain elongation. This increase is also the cause of dynamic grain growth in the material. The larger the grains, the more difficult it becomes to provide this sliding mechanism. This phenomenon expresses itself as strain hardening.

The set of stress-strain curves, as mentioned in Section 2.1 , is derived from the force-displacement data of the tensile experiments, by means of inverse modelling. This is an iterative process in which tabular stress-strain data at different strain rates converge to a state where the simulation results match the experimental results as closely as possible.

To fit the hardening part of the curves into a phenomenological model, the Voce hardening law is used, according to Equation (2).

$$
\sigma_{\mathrm{h}}=\sigma_{\mathrm{y} 0}+\Delta \sigma\left[1-\exp \left(-\frac{\varepsilon}{\varepsilon_{0}}\right)\right]
$$

where $\Delta \sigma$ is the saturation stress, and $\varepsilon_{0}$ is a reference strain. From the tensile experiments, these two constants have to be determined.

Another option would be the use of Nadai hardening, with a power law, but because of the very high failure strain, this hardening law is not applicable to superplastic aluminium [2].

\subsection{SOFTENING}

Superplastic materials fail when cavities inside the material coalesce with eachother, thereby showing a strong decrease in the macroscopic flow stress. Before coalescence takes place, cavities nucleate and grow, which already can show softening behaviour. From non-destructive tensile tests, the void volume fraction $\xi$ was observed, which turned out to be a function of the plastic strain only (and not of the plastic strain rate). This response is also reported in [3], and can be considered a bilinear relationship. Three parameters have to be determined in this case: two slopes $c_{1}$ and $c_{2}$ and a critical plastic strain $\varepsilon_{\mathrm{c}}$, where the $\xi-\varepsilon$ relation changes slope from the lower $c_{1}$ to the higher $c_{2}$.

The void volume fraction influences the macrosopic stress $\sigma_{\mathrm{h}}$, according to Equation (3)

$$
\sigma_{\mathrm{s}}=\sigma_{\mathrm{h}}\left(1-\eta_{1} \xi^{\eta_{2}}\right)^{\eta_{3}}
$$

The parameters $\eta_{1}, \eta_{2}$ and $\eta_{3}$ are material constants and must be determined in order to fit this equation to the stress-strain curves, especially in the softening part.

\subsection{BACKPRESSURE INFLUENCE}

Since superplastic materials show a very low flow stress compared to conventional metal plasticity, it is possible to slow down the cavity nucleation and growth by applying an external pressure to the material during the forming process [4]. It is therefore possible to reach even higher plastic strains before failure if this backpressure is applied. This behaviour can be observed by performing biaxial experiments.

Gas leak through the formed sheet is a measure for failure. Figure 2 shows the results of leak measurements by applying an overpressure of 1 bar He on the inside of the bulge. As is clearly visible, bulges formed with a higher backpressure show less permeability for the gas at the same bulge height. Also, the higher the backpressure, the higher the maximum bulge height.

\subsection{BIAXIAL FLOW BEHAVIOUR}

In [5], it is concluded that Al-Mg alloys at high temperature do not conform to the von Mises or Hill flow criteria. The flow criterium of Logan-Hosford is much more suitable and can be seen as a generalisation of the von Mises flow criterium. The Lankford strain ratio $R$ can be taken into account, resulting in Equation 4 for planes stress situations, where the equivalent stress can be written as

$$
\sigma_{\mathrm{f}}^{m}=\frac{1}{1+R}\left(\sigma_{1}^{m}+\sigma_{2}^{m}\right)+\frac{R}{1+R}\left(\sigma_{1}-\sigma_{2}\right)^{m}
$$

where $\sigma_{1}$ and $\sigma_{2}$ are the in-plane principal stresses. The exponent $m$, having the value of 2 in case of von Mises 


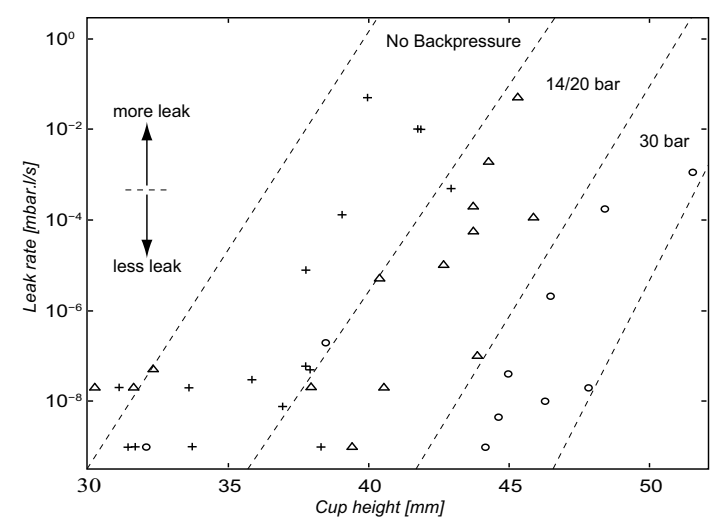

Figure 2: Leak rate of the formed cups as function of the cup heigth in free bulging. The cup diameter is $70 \mathrm{~mm}$

flow, is proposed to have a value of 8 for fcc metals. The Lankford strain ratio can be determined from tensile experiments, by measuring width and thickness of the tensile bars after non-destructive testing (no necking). Table 2 shows $R$ in different directions with respect to the rolling direction. It is clear, from the value of $\Delta R$ that the material can be considered in-plane isotropic.

Table 2: Lankford strain ratios of ALNOVI-1

\begin{tabular}{ccccc}
\hline$R_{0}$ & $R_{45}$ & $R_{90}$ & $R$ & $\Delta R$ \\
\hline 0.816 & 0.827 & 0.829 & 0.825 & -0.0043 \\
\hline
\end{tabular}

\section{SUPERPLASTIC MATERIAL MODEL}

The standard built-in material models in ABAQUS are not sufficient to represent the superplastic material behaviour which is necessary here. With a user-defined material model, the Hosford yield criterion can be incorporated, together with the influence of a backpressure on the forming behaviour. First, the routine consists of a part where the elastic properties are defined. Secondly, an algorithm is necessary to determine whether the material is in this elastic state or if it will behave plastically. If so, then the stress update algorithm calculates the stress tensor, followed by a routine where plastic strains and state variables are updated.

\subsection{ELASTIC PROPERTIES}

The elastic part of the user subroutine is used to calculate the stress tensor from the strain increment input. In case of superplastic sheet forming simulations, a plane stress formulaton is used to calculate the trial stress vector (stresses and strains are represented in vector notation in ABAQUS). Since the Hosford equivalent stress $\sigma^{\text {eq }}$ uses principal stresses for its calculation, these principal values have to be calculated first, using a utility subroutine in ABAQUS. The equivalent stress can then be calculated using Equation (4).

\subsection{FLOW STRESS}

To determine if $\sigma^{\text {eq }}$ falls inside the elastic or plastic range at increment $\mathrm{t}_{1}$, a flow stress has to be calculated. Equations (1) to (3) are therefore used with a value for the equivalent plastic strain $\bar{\varepsilon}^{\mathrm{p}}$, calculated in increment $\mathrm{t}_{0}$, and an equivalent plastic strain rate $\dot{\bar{\varepsilon}}^{\mathrm{p}}$ equal to zero.

The backpressure $p$ also has an influence on the flow stress. This backpressure is a user-defined field in ABAQUS, which is read by the user subroutine of the material model. An extra parameter $c_{3}$ is used which has the effect that the critical plastic strain $\varepsilon_{\mathrm{c}}$ in increased as function of $p$. The void volume fraction $\xi_{\mathrm{c}}$ belonging to this backpressure-dependent $\varepsilon_{\mathrm{c}}$ is a constant. The slopes $c_{1}$ and $c_{2}$ are altered to $c_{1}^{*}$ and $c_{2}^{*}$ according to Equation (5) and $\varepsilon_{\mathrm{c}}$ is altered to $\varepsilon_{\mathrm{c}}^{*}$ according to Equation (6)

$$
\begin{gathered}
c_{1,2}^{*}=\frac{c_{1,2}}{1+c_{3} p} \\
\varepsilon_{\mathrm{c}}^{*}=\frac{\varepsilon_{\mathrm{c}}}{1+c_{3} p}
\end{gathered}
$$

\subsection{STRESS UPDATE}

A Newton iteration scheme is used in which the increase in equivalent plastic strain and void volume fraction is calculated. Also the flow stress is incorporated in this iteration scheme. With the updated equivalent plastic strain increment, the incremental plastic strain vector can be determined, if the flow direction $\partial f / \partial \sigma$ is known. The two limiting situations in determining this direction are:

- calculation in the current time increment $\partial f / \partial \sigma^{\left(\mathrm{t}_{1}\right)}$. A drawback of this situation is that the return direction vector does not intersect with the yield surface as calculated from the equivalent plastic strain and strain rate, calculated in the current time increment;

- as calculated from the stress vector of the previous increment $\partial f / \partial \sigma^{\left(t_{0}\right)}$. This conforms to an explicit scheme, so it is bound to a maximum time increment in order to keep accuracy.

A factor $\alpha$ can be introduced to weigh the influence of both options, according to Equation (7)

$$
\frac{\partial f}{\partial \sigma}=\alpha \frac{\partial f}{\partial \sigma^{\left(\mathrm{t}_{1}\right)}}+(1-\alpha) \frac{\partial f}{\partial \sigma^{\left(\mathrm{t}_{0}\right)}}
$$

An estimate for $\alpha$ can be based on the values of the equivalent trial stress $\sigma_{\text {eq }}^{\left(\mathrm{t}_{1}\right)}$, the equivalent stress of the stress vector from the previous increment $\sigma_{\mathrm{eq}}^{\left(\mathrm{t}_{0}\right)}$, and the yield stress as calculated in the stress update algorithm, $\sigma_{\mathrm{y}}^{\left(\mathrm{t}_{1}\right)}$. This proportional estimate is according to Equation (8)

$$
\alpha=\frac{\sigma_{\mathrm{y}}^{\left(\mathrm{t}_{1}\right)}-\sigma_{\mathrm{eq}}^{\left(\mathrm{t}_{0}\right)}}{\sigma_{\mathrm{eq}}^{\left(\mathrm{t}_{1}\right)}-\sigma_{\mathrm{eq}}^{\left(\mathrm{t}_{0}\right)}}
$$




\section{SIMULATIONS}

The user-defined material as developed according to the previous section is tested by simulating the bulge experiments. Features that can be compared with the experiments are the resulting bulge height and the sheet thickness in the top of the bulge. The undeformed specimens were rasterised by a grid. This gives an indication of the stretch of the material after deforming, especially on the top of the bulge. This feature will also be compared with the simulations. After evaluation of the model, some considerations are mentioned in case of the forming simulation of a RF shield.

\subsection{BULGE TEST SIMULATIONS}

The bulge experiments were divded into three target strain rates and four backpressures. The target strain rates, which are the maximum strain rate in the model during deformation, is forced by a precalculated pressure progress in time: 6,12 and $18 \cdot 10^{-4} \mathrm{~s}^{-1}$ [6]. The backpressures used are $0,14,22$ (20 at an initial sheet thickness of 1.0 $\mathrm{mm}$ ) and 30 bar. Two initial sheet thicknesses has been used, 0.8 and $1.0 \mathrm{~mm}$.

The results of one of the simulations are presented for the target strain rate of $12 \cdot 10^{-4} \mathrm{~s}^{-1}$ in Figure 3, where a backpressure of 30 bar has been applied. The initial sheet thickness is $0.8 \mathrm{~mm}$. The results show that the analysis underestimates the top thickness of the bulge slightly.

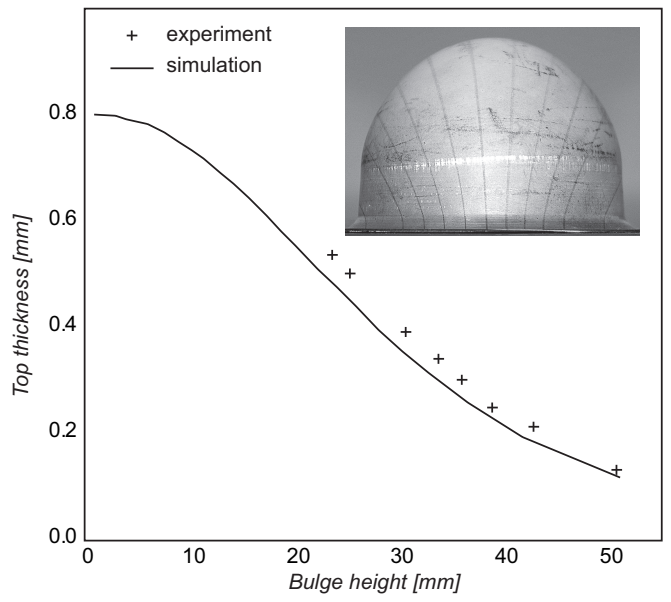

Figure 3: Results of the simulation of the bulge forming process compared with the experiment

\subsection{RF SHIELD SIMULATION}

The material model as it is constructed in order to perform superplastic forming simulations, needs some addition where it comes to the prediction of gas leak through a formed sheet. The first problem is the fact that the measured gas leakage has a high standard deviation. This makes it difficult to predict the absolute value of the gas leak. A possible solution is that not this value, but a value for a leak risk is implemented. A probability will then be calculated which is the chance of not conforming to the leak risk constraint. The second problem is the coupling of the leak (risk) to the existing output of the simulation. It seems straightforward to couple the leak to the equivalent plastic strain or void volume fraction, but also the initial sheet thickness can possibly influence this value. A third problem is the implementation of friction with respect to the gas leak, it is not trivial that a higher friction coefficient will negatively influence the gas leak value at higher plastic strains.

\section{CONCLUSIONS}

The results of the simulations show that the superplastic forming process can be described in a user-defined material model by an initial flow equation, a Voce hardening model and a softening part caused by void nucleation and growth. A backpressure can be incorporated by forcing a higher value for the critical strain, where the void volume fraction starts growing more rapidly (transition from slope $c_{1}$ to $c_{2}$ ).

Since the leak value is a measure for failure, the next step will be the addition of a leak value or risk into the material model. This value has a relatively high standard deviation, and must be inserted with great care, since leak is the most important constraint in case of RF shields in particle detectors. Bulge experiments with a die must give information on the frictional behaviour of the material and the influence of the friction on the gas leak.

\section{REFERENCES}

[1] R.A. Vasin, F.U. Enikeev, M.I. Mazurski, and O.S. Munirova. Mechanical modelling of the universal superplastic curve. Journal of Materials Science, 35 : 2455-66, 2000.

[2] A.H. Van Den Boogaard. Thermally Enhanced Forming of Aluminium Sheet. $\mathrm{PhD}$ thesis, University of Twente, November 2002.

[3] S.L. Semiatin, V. Seetharaman, A.K. Ghosh, E.B. Shell, M.P. Simon, and P.N. Fagin. Cavitation during hot tension testing of ti-6al-4v. Materials Science and Engineering A, pages 92-110, 1998.

[4] B. Davis and J. Hryn. Innovative forming and fabrication technologies: New opportunuities. Technical report, Argonne National Laboratory, December 2007.

[5] T. Naka, Y. Nakayama, T. Uemori, R. Hino, and F. Yoshida. Effects of temperature on yield locus for 5083 aluminium alloy sheet. Journal of Materials Processing Technology, 140:494-9, 2003.

[6] S.G. Luckey Jr., P.A. Friedman, and K.J. Weinmann. Correlation of finite element analysis to superplastic forming experiments. Journal of Materials Processing Technology, 194:30-7, 2007. 